\title{
Proving the triviality of rational points on Atkin-Lehner quotients of Shimura curves
}

\author{
Pierre Parent and Andrei Yafaev
}

\begin{abstract}
In this paper we give a method for studying global rational points on certain quotients of Shimura curves by Atkin-Lehner involutions. We obtain explicit conditions on such quotients for rational points to be "trivial" (coming from CM points only) and exhibit an explicit infinite family of such quotients satisfying these conditions.
\end{abstract}

\section{Introduction.}

The study of points on Shimura curves rational over number fields or, more precisely, finding families of such curves whose only rational points are special (or CM) is an interesting problem for several reasons. One reason is that these curves may provide non-trivial examples of violation of the Hasse principle accounted for by the Brauer-Manin obstruction. This question was the main motivation for [31], [27], [29] and [30].

Another motivation is the question of scarcity of possible endomorphism algebras of abelian varieties over $\mathbb{Q}$ of $\mathrm{GL}_{2}$-type. Let us explain this in more detail, following the introduction to [5]. One says that an abelian variety $A$ over $\mathbb{Q}$ is of $\mathrm{GL}_{2}$-type if the endomorphism algebra $\mathbb{Q} \otimes \operatorname{End}_{\mathbb{Q}}(A)$ is a number field of degree $\operatorname{dim}(A)$. In loc. cit. the authors formulate the following conjecture (attributed to Coleman, also made by Clark and Mazur): for $g$ any positive integer, the number of isomorphism classes of $\overline{\mathbb{Q}}$-endomorphism algebras of $g$-dimensional abelian varieties of $\mathrm{GL}_{2}$-type is finite. We refer to loc. cit. for more details on this and just mention that the "GL $\mathrm{L}_{2}$-type" hypothesis in the conjecture is motivated by a conjecture of Ribet predicting that abelian varieties over $\mathbb{Q}$ of $\mathrm{GL}_{2}$-type are modular: in the latter case, the extended experimental knowledge available on modular abelian varieties gives evidence supporting the conjecture. Note also that, as Ribet himself has shown, his conjecture follows from Serre's conjecture on modularity of $\bmod p$ representations, and that a proof of this conjecture has recently been announced by Khare et al. For $g=1$, the theory of complex multiplication, plus the classical result that there 
is only a finite number of quadratic imaginary orders with class number 1, show that Coleman's conjecture is true. For $g \geq 2$ very little is known. When $g=2$ the endomorphism algebras that may occur are orders in either $M_{2}(\mathbb{Q})$, or $M_{2}(K)$ for $K$ an imaginary quadratic field (in these cases the abelian surfaces are geometrically reducible), or (in the irreducible case) a quadratic field or an indefinite division quaternion algebra over $\mathbb{Q}$ (see [5], introduction and references therein).

The diophantine study of Shimura curves allows to address the question of excluding this latter case of (potential) quaternionic multiplication, as these curves are coarse moduli spaces for abelian surfaces endowed with a geometric action of some order in a quaternion algebra. Indeed if $D$ is an integer which is the product of an even number of distinct primes and $m$ is a square-free integer, we say that the pair $(D, m)$ is of $\mathrm{GL}_{2}$-type if there is an abelian surface $A$ over $\mathbb{Q}$ such that $\operatorname{End}_{\mathbb{Q}}(A) \otimes \mathbb{Q}=\mathbb{Q}(\sqrt{m})$ and $\operatorname{End}_{\overline{\mathbb{Q}}}(A) \otimes \mathbb{Q}=B_{D}$ (the quaternion algebra ramified at primes dividing $D$ ). Let $X^{D}$ be the Shimura curve over $\mathbb{Q}$ attached to a maximal order in $B_{D}$ (denoted by $X_{D}$ in [5]), and if $n$ is a divisor of $D$, let $w_{n}$ be the Atkin-Lehner involution associated to $n$. Recall that a point on these curves is said to be a special (or CM) point if the underlying abelian surface $A$ is isogenous to a square of an elliptic curve having complex multiplication by a field $K$ (in which case $\operatorname{End}_{\overline{\mathbb{Q}}}(A) \otimes \mathbb{Q} \simeq M_{2}(K)$ contains any rational quaternion algebra split over $K$ ). With the above notations Rotger proves the following result: if $(D, m)$ is a pair of $\mathrm{GL}_{2}$-type over $\mathbb{Q}$, then $m \mid D$ and $X^{D} / w_{m}(\mathbb{Q})$ is non-trivial, i.e. contains a non-special point (see [25], Theorem 4.4).

It has long been known that $X^{D}(\mathbb{Q})$ is empty (in fact $X^{D}(\mathbb{R})=\emptyset$ ). More generally the results of Jordan, Livné, Ogg, Shimura and some others give a description of the set of local points on Shimura curves and their Atkin-Lehner quotients. Their results provide necessary conditions for $X^{D} / w_{m}$ to have local points everywhere, and this leads to the following (see [27], Theorem 3.1):

Theorem 1.1 If $X^{D} / w_{m}(\mathbb{Q}) \neq \emptyset$, then either $m=D$ or $m=D / p$ for some $p$ dividing $D$. Furthermore if $(D, m)=(p q, q)$ for two odd primes $p$ and $q$ then $\left(\frac{q}{p}\right)=-1$ and either:

1. $p \equiv 3 \bmod 4$, or

2. $p \equiv 1 \bmod 4$ and $q \equiv 3 \bmod 4$.

The case $m=D$ is one of the main questions addressed by P. Clark in [6]. Unfortunately, the method we develop in this paper does not work in this case. The reason is that our method relies, in a crucial way, on the existence of a non-trivial rank zero quotient of the jacobian of the curve. Considerations of the sign of the functional equation of $L$-functions show that the jacobian of 
the curve $X^{D} / w_{D}$ has no non-trivial rank-zero quotient (at least if one believes in the Birch and Swinnerton-Dyer conjecture).

However in the case where $m=D / p$, there is no obstruction to the existence of such a quotient coming from the sign of the functional equation. Let $X$ be the curve $X^{D} / w_{m}$. We are interested in determining whether $X(\mathbb{Q})$ is "trivial" (in the sense that it consists only of special points). Our strategy follows the lines of the paper [22] of the first author, with several new ingredients. More precisely a local study as in [21], section 1, leads to considering two subcases that Ogg calls "ramifié" and "non-ramifié" (see loc. cit., p. 204 et seq.) that correspond respectively to the two cases at the end of the above theorem. For technical reasons it is easier to push our methods further in the latter ("non-ramifié") case, and we limit ourselves to this case in this paper. For a given pair $(p q, q)$ one can in principle verify, by computation, whether our conditions are satisfied. However in this article we were not able to make our method work for infinitely many $q$ s. We nevertheless conclude our paper by exhibiting an infinite family of AtkinLehner quotients of Shimura curves whose only possible rational points are trivial in the above sense, although these curves may have local points at every prime of bad reduction. (Actually, one can also check that in our family, there are no $\mathbb{Q}$-rational special points either, so the set of rational points is empty; see section 6). As far as we know, this was previously known for finitely many curves only (but see also Rotger's result discussed below).

We close this introduction by discussing a recent result, similar to the one obtained in this article. After the present paper was completed we learned from V. Rotger that he could also prove the triviality of rational points in infinite families of Atkin-Lehner quotients of Shimura curves, but in the "ramifié" case quoted above, more precisely for pairs $(p q, q)$ as in the first case of Theorem 1.1 (see [26], Theorem 1.2). His methods are totally different from ours: he uses the modular interpretation of the points on the Shimura curves and analyzes the Galois representations provided by torsion points of the corresponding abelian surfaces. Note that our results are complementary as we focus on the "non-ramifié" case. In fact, as Rotger himself pointed out to us, it follows from his work that in this latter case the field of definition of modular points is different from the field of moduli, i.e. the $\mathbb{Q}$-valued points correspond to abelian surfaces that cannot be defined over $\mathbb{Q}$. On the other hand, our methods do not use the modular interpretation of the points, so they cover cases that cannot be tackled by Rotger's techniques.

\subsection{The strategy.}

We now sketch our method. Suppose that $D$ is a product of two primes $D=p q$ and $m$ is $q$. Let $X:=X^{p q} / w_{q}$ be as above and suppose that there is a point $P$ in $X(\mathbb{Q})$. Assume that we are in the case 2 of Theorem 1.1. Consider the model of $X$ over $\mathbb{Z}_{p}$ given by the Cherednik-Drinfeld 
theorem and let $\tilde{X}$ be the regular model of $X$ obtained by blowing-up at singular points. Looking at the specialization of $P$ in $\tilde{X}$ at $p$, one sees as in [21], p. 205, that $P$ specializes precisely to an exceptional component coming from a blow-up of a singular point of width 2 in the special fiber of $X$, i.e. a singular point having "multiplication by $i$ ". The same is true for $w_{p}(P)$, therefore the specializations of our two rational points $P$ and $w_{p}(P)$ belong to the smooth locus of the same exceptional component at $p$. Let $\phi_{P}$ be the Albanese embedding of $X_{/ \mathbb{Z}_{p}}^{\mathrm{sm}}$ into the Néron model of its jacobian $J$ defined by $\phi_{P}(Q)=(Q)-(P)$. Let $X_{p+1, \mathbb{Q}}$ be the curve $(p+1) \phi_{P}\left(X_{\mathbb{Q}}\right)$ in $J_{\mathbb{Q}}$.

Assuming that $p$ is much larger than $q$ we prove that $X_{p+1, \mathbb{Q}}$ has, at least locally, a smooth model around $0_{\mathbb{F}_{p}}$, and the points $(p+1) \phi_{P}(P)$ and $(p+1) \phi_{P}\left(w_{p}(P)\right)$ coincide in $X_{p+1}\left(\mathbb{F}_{p}\right)$. This is the content of sections 2 and 3. We hope that the description we give here of the asymptotic shape (as the discriminant increases) of the graph of special fibers of Shimura curves, and therefore of the component groups of their jacobians, can be of independent interest.

Next we consider the natural map $\Psi_{P}$ from $X$ to the "winding quotient" $J_{e}$ of $J$. We prove as in [22] that, if one can find on the graph of $X$ a closed path "made" of Gross-Heegner vectors containing the component of $P\left(\mathbb{F}_{p}\right)$, then $\Psi_{P}$ is a formal immersion at our point $P_{\mathbb{F}_{p}}$. This is proved in section 5, using the results of section 4. As $p+1$ is prime to $p$, the same is true for the map from $X_{p+1}$ to $J_{e}$ at $(p+1) \phi_{P}(P)_{\mathbb{F}_{p}}$. The classical argument of Mazur's method now applies, giving that $(p+1) \phi_{P}(P)=(p+1) \phi_{P}\left(w_{p}(P)\right)$ in $X_{p+1}(\mathbb{Q})$, so $(p+1)\left((P)-\left(w_{p}(P)\right)\right)=0$ in $J(\mathbb{Q})$. This means that either $P=w_{p}(P)$, in which case $P$ is indeed a special point, or $X$ has gonality less than $p+1$. If $q$ is larger than 245 this contradicts Abramovich's bound on the gonality of Shimura curves, and this argument concludes the proof (see Theorem 5.3).

One sees from the above sketch that one of the main problems is to construct a "closed path made of Gross-Heegner vectors containing the component of $P\left(\mathbb{F}_{p}\right)$ on the graph of $X$ ". This is what we do in the last section for a fixed $q=251$, and $p$ ranging through an infinite set of primes satisfying certain congruences.

Acknowledgments.

We are grateful to Bas Edixhoven, Qing Liu, Victor Rotger and Emmanuel Ullmo for instructive discussions on the subject of this paper. We thank the referee for useful comments and suggestions.

\section{Admissible curves and their jacobians.}

We start by recalling the notion of admissible curve in the sense of Jordan and Livné, and some of their basic properties. The theorem of Cherednik and Drinfeld implies that the Shimura curves 
and their Atkin-Lehner quotients have natural models which are admissible curves at primes dividing their discriminant. For more details we refer to [17], section 3, or [24], section 2.

Let $p$ be a prime number and $K$ a $p$-adic field with ring of integers $\mathcal{O}$, uniformizer $\pi$ and residue field $k$. Let $X$ be a proper and flat curve over $\mathcal{O}$. We say that $X$ is admissible if its generic fiber $X_{K}$ is smooth over $K$, its special fiber $X_{k}$ is potentially (i.e., after a finite extension of $k$ ) a union of projective lines with reduced normal crossings, and each singularity of $X_{k}$ has a local equation $x y=\pi^{e}$ where $e \geq 1$ is some integer called the width of the singularity. The curve $X$ is regular at a singular point if and only if the width of this point is 1 . From an arbitrary admissible curve $X$ one obtains a regular one $\tilde{X}$ by making successive blow-ups at the singular points. This leads to replacing a singularity of width $e$ by a chain of $e-1$ projective lines whose intersections points have width 1 . We write $X^{\mathrm{sm}}$ for the largest open subset of $X$ which is smooth over $\mathcal{O}$.

The fiber $X_{k}$ of an admissible curve is combinatorially described by its dual graph, which we denote by $\mathcal{G}\left(X_{k}\right)$. Recall that this is the unoriented graph whose vertices $\mathcal{V}$ are the components of $X_{k}$, and whose edges $\mathcal{E}$ are the singular points of $X_{k}$ : an edge links two vertices if the corresponding singular point is the intersection point of the two corresponding components. One endows each edge with a length, which is defined to be the width of the corresponding singularity.

The neutral component $\operatorname{Pic}^{0}\left(X_{k}\right)$ of the Picard scheme of $X_{k}$ is a torus whose character group is isomorphic to the homology group $H_{1}\left(\mathcal{G}\left(X_{k}\right), \mathbb{Z}\right)$. It follows from the above that the regularization of $X_{k}$ amounts to replacing each edge of length $e$ in $\mathcal{G}\left(X_{k}\right)$ by a chain of $e$ edges of length 1 . This shows that $H_{1}\left(\mathcal{G}\left(X_{k}\right), \mathbb{Z}\right)$ and $H_{1}\left(\mathcal{G}\left(\tilde{X}_{k}\right), \mathbb{Z}\right)$ are canonically isomorphic. There is a canonical Euclidean pairing (the monodromy pairing) on $H_{1}\left(\mathcal{G}\left(X_{k}\right), \mathbb{Z}\right)$ which is deduced from the pairing on $\mathbb{Z}^{\mathcal{E}}$ defined by $\left\langle e_{i}, e_{j}\right\rangle=\operatorname{width}\left(e_{i}\right)^{-1} \delta_{i, j}$ (here $\delta$ denotes the Kronecker symbol). The following proposition explicits the isomorphism $\operatorname{Pic}^{0}\left(X_{k}\right) \simeq H_{1}\left(\mathcal{G}\left(X_{k}\right), \mathbb{Z}\right) \otimes \mathbb{G}_{m}$ of [15], 12.3.7.

Proposition 2.1 Let $X$ be an admissible curve over $\mathcal{O}$ as above and let $X_{\bar{k}}$ be its special fibre. Let $C_{0}$ be a component of $X_{\bar{k}}$, and $H$ be the subgroup of $\operatorname{Pic}^{0}\left(X_{\bar{k}}\right)$ consisting of linear equivalence classes of Weil divisors of degree zero on $X_{\bar{k}}$ whose support is contained in the smooth locus $C_{0}^{\mathrm{sm}}$ of $C_{0}$. Let $\left\{\mathcal{C}_{i}\right\}, i=1, \ldots, n$ be the set of connected components of $X_{\bar{k}} \backslash C_{0}^{\mathrm{sm}}$. For each $i$ let $A_{i j}, j=1, \ldots, n_{i}$ be the set of singular points of $C_{0} \cap \mathcal{C}_{i}$. Let $x$ be a coordinate function on the rational curve $C_{0}$.

There is a $\bar{k}$-isomorphism from $\operatorname{Pic}^{0}\left(X_{\bar{k}}\right)$ to $T_{1} \times \cdots \times T_{n} \times \mathcal{T}$, where the $T_{i}$ 's and $\mathcal{T}$ are tori with $\operatorname{dim}\left(T_{i}\right)=n_{i}-1$, such that any element $\sum_{r}\left(P_{r}\right)-\sum_{r}\left(Q_{r}\right)$ of $H$ is mapped to $\left(\left(\prod_{r} \frac{x\left(A_{i 1}\right)-x\left(Q_{r}\right)}{x\left(A_{i j}\right)-x\left(Q_{r}\right)} \cdot \frac{x\left(A_{i j}\right)-x\left(P_{r}\right)}{x\left(A_{i 1}\right)-x\left(P_{r}\right)}\right)_{2 \leq j \leq n_{i}}\right)_{1 \leq i \leq n} \times 1$ in $\left(\prod_{i=1}^{n} T_{i}(\bar{k})\right) \times \mathcal{T}(\bar{k})$. 
Proof. Let $D$ be a divisor on $X_{\bar{k}}$. Let $f_{D}$ be a function on the normalization of $X_{\bar{k}}$ having divisor $D_{\mid C}$ on each component $C\left(\simeq \mathbb{P} \frac{1}{k}\right)$. Let $\mathcal{B}$ be a basis of $H_{1}\left(\mathcal{G}\left(X_{\bar{k}}\right), \mathbb{Z}\right)$. One associates to $D$ the element $\left(\prod_{h_{i}, h=\sum h_{i}}\left(f_{D}\left(s\left(h_{i}\right)\right) / f_{D}\left(t\left(h_{i}\right)\right)\right)\right)_{h \in \mathcal{B}}$ of $H_{1}\left(\mathcal{G}\left(X_{\bar{k}}\right), \mathbb{Z}\right) \otimes \mathbb{G}_{m}$, where the symbol $h=\sum h_{i}$ denotes the path $h$ consisting of successive edges $h_{i}$, and $s\left(h_{i}\right), t\left(h_{i}\right)$ denote the points of $X_{\bar{k}}$ corresponding to the source and target of $h_{i}$ respectively (with respect to some fixed orientation on the graph).

As $H_{1}\left(\mathcal{G}\left(X_{\bar{k}}\right), \mathbb{Z}\right)=\oplus_{1 \leq i \leq n} H_{1}\left(\mathcal{G}\left(\mathcal{C}_{i} \cup C_{0}\right), \mathbb{Z}\right)$, we can suppose without loss of generality that $n=1$. We have $H_{1}\left(\mathcal{G}\left(X_{\bar{k}}\right), \mathbb{Z}\right)=H_{1}\left(\mathcal{G}^{\prime}, \mathbb{Z}\right) \oplus H_{1}\left(\mathcal{G}\left(\mathcal{C}_{1}\right), \mathbb{Z}\right)$ where $\mathcal{G}^{\prime}$ is the graph of some sub-curve $C^{\prime}$ of $X_{\bar{k}}$, and this splitting induces a decomposition $\operatorname{Pic}^{0}\left(X_{\bar{k}}\right) \simeq H_{1}\left(\mathcal{G}^{\prime}, \mathbb{Z}\right) \otimes \mathbb{G}_{m} \times H_{1}\left(\mathcal{G}\left(\mathcal{C}_{1}\right), \mathbb{Z}\right) \otimes \mathbb{G}_{m}$. The subgroup $H$ is mapped isomorphically to the first factor, and has trivial image in the second. Indeed, as above, we associate to an element $D=\sum_{r}\left(P_{r}\right)-\left(Q_{r}\right)$ of $H$ a function $f_{D}$ on $X_{\bar{k}}$ defined by $f_{D} \equiv 1$ on the complement of $C_{0}$ and $f_{D}(x)=\prod_{r} \frac{x-x\left(P_{r}\right)}{x-x\left(Q_{r}\right)}$ on $C_{0}$. The map sending $D$ to $\left(f_{D}\left(A_{1, j}\right) / f_{D}\left(A_{1,1}\right)\right)_{2 \leq j \leq n_{1}}$ identifies $H$ with $\mathbb{G}_{m}^{n_{1}-1}$.

This proposition describes the Albanese images of an admissible curve in its jacobian. We are now interested in models of the multiples of these images. More precisely, letting $X$ be an admissible curve over $\operatorname{Spec}(\mathcal{O})$, we let $J_{K}:=\operatorname{Jac}\left(X_{K}\right)$ and $J$ be its Néron model over $\operatorname{Spec}(\mathcal{O})$. Let $P$ and $P_{0}$ be two points of $X(\mathcal{O})$. Call $\phi: X^{\text {sm }} \rightarrow J$ the Albanese embedding defined by $\phi(Q):=(Q)-\left(P_{0}\right)$. For any integer $n$ the image of $X_{K}$ by $n \cdot \phi_{K}$ in the generic fiber of $J$ is a proper curve over $K$ which we call $X_{n, K}$. Following [11], section 9, we say that a point on an admissible curve is non-disconnecting if the special fiber of the curve deprived from this point remains connected.

Proposition 2.2 Assume the following:

1. the points $P$ and $P_{0}$ specialize to the same component $C_{0}$ of $X_{k}$;

2. the component $C_{0}$ intersects other components at two singular points only, which are nondisconnecting;

3. $\phi\left(X_{K}\right) \cap J_{K}[n]=\{0\}$;

4. the image of $X$ by $\phi$ has no non-trivial $n$-torsion in the component group of $J$, i.e. if $C \neq C_{0}$ is a component of $X_{k}$ then $n \cdot \phi\left(C^{\mathrm{sm}}\right) \nsubseteq J_{k}^{0}$.

Then there is a smooth model of $X_{n, K}$ over $\mathcal{O}$, i.e. a smooth $\operatorname{Spec}(\mathcal{O})$-scheme $X_{n}$ of relative dimension 1 such that $X_{n} \times_{\operatorname{Spec}(\mathcal{O})} \operatorname{Spec}(K)=X_{n, K}$, which contains $n \cdot \phi(P)(\operatorname{Spec}(\mathcal{O}))$ and $n \cdot \phi\left(P_{0}\right)(\operatorname{Spec}(\mathcal{O}))$. 
Proof. Let $J^{0}$ be the neutral component of $J$ and let $\operatorname{Pic}^{0}(X)$ be the usual functor from the category of $\operatorname{Spec}(\mathcal{O})$-schemes to the category of groups which associates to $S$ the group of invertible sheaves having degree 0 on every component of $X \times_{\operatorname{Spec}(\mathcal{O})} S$. As $X$ is semistable over $\mathcal{O}$ the scheme $J^{0}$ is semiabelian, so it represents $\operatorname{Pic}^{0}(X)$ (see [23], proof of Théorème 2, $i)$ ). Therefore the assumption 1 implies that $J^{0}$ contains $\phi(P)$ and $\phi\left(P_{0}\right)=0$. Let $X_{n}$ be the scheme-theoretic closure of $n \cdot \phi(X)_{K}$ in $J^{0}$, i.e. the smallest closed subscheme (with reduced induced structure) of $J^{0}$ containing $n \cdot \phi(X)_{K}$. Let $A_{1}$ and $A_{2}$ be the singular points of $C_{0}$. Proposition 2.1 says that for $Q \in C_{0}(\bar{k}) \backslash\left\{A_{1}, A_{2}\right\}$,

$$
\phi(Q)=\lambda\left(x(Q)-x\left(A_{1}\right)\right) /\left(x(Q)-x\left(A_{2}\right)\right) \times 1
$$

in a suitable decomposition $\operatorname{Pic}^{0}\left(X_{\bar{k}}\right) \simeq T_{1} \times \mathcal{T}$ (with $\lambda=\left(x\left(P_{0}\right)-x\left(A_{2}\right)\right) /\left(x\left(P_{0}\right)-x\left(A_{1}\right)\right)$ ). One deduces from that and the assumption 2 that the special fibre of $X_{n}$ contains the same onedimensional subtorus $T_{1}$ of $J_{k}^{0}$ as the image of the component $C_{0}^{\text {sm }}$ of $X$. By [14], Proposition 2.8.1, $X_{n}$ (which is a scheme-theoretic closure) is flat over $\operatorname{Spec}(\mathcal{O})$. The assumption 3 insures that $X_{n}$ is smooth over $K$. To prove the smoothness of $X_{n}$ over $\mathcal{O}$ it is therefore enough to prove that its special fiber is smooth over $k$, and this in turn follows from the assumption 4.

\section{Bad reduction of Shimura curves.}

In this section we specialize the above considerations to the case of Shimura curves. We begin by recalling some basic facts. Let $D$ be a product of an even number of distinct (finite) primes, let $N$ be a integer prime to $D$, and $\mathcal{O}_{0}^{D}(N)$ an order of level $N$ in the quaternion algebra $B_{D}$ over $\mathbb{Q}$ of discriminant $D$. As $B_{D}$ is indefinite (i.e. $\mathbb{R} \otimes B_{D}$ is split), there is an embedding of the subgroup of $\mathcal{O}_{0}^{D}(N)^{\times}$consisting of elements with norm 1 into $\mathrm{SL}_{2}(\mathbb{R})$, and the quotient of the Poincaré upper half-plane by this subgroup via the usual homographic action is a compact Riemann surface called the Shimura curve associated to $\mathcal{O}_{0}^{D}(N)$. We denote this curve by $X_{0}^{D}(N)(\mathbb{C})$. It follows from the work of Shimura that it has a canonical model over $\mathbb{Q}$, and thanks to Deligne-Rapoport and Buzzard (model at primes dividing the level $N$ ), Cherednik-Drinfeld et al. (model at primes dividing the discriminant $D$ ) one has a model for $X_{0}^{D}(N)$ over $\mathbb{Z}$. This model is constructed as the coarse moduli scheme parametrizing abelian surfaces with multiplication by $\mathcal{O}_{0}^{D}(N)$, i.e. abelian varieties of dimension 2 with multiplication by a maximal order of $B_{D}$ and a compatible $N$-level structure (plus an additional technical assumption in characteristics dividing $D$ that we will not discuss here). We will only need the description of Cherednik-Drinfeld, i.e. we will concentrate on the localization of $X_{0}^{D}(N)$ at primes dividing $D$. Fix such a prime $p$. To simplify the notations, set $X:=X_{0}^{D}(N)$. According to Cherednik and Drinfeld, $X$ is an admissible curve 
over $\mathbb{Z}_{p}$. Let $\mathcal{G}:=\mathcal{G}\left(X_{\mathbb{F}_{p}}\right)$ be the dual graph of $X_{\mathbb{F}_{p}}$ as in section 2 . This graph is easier to describe when $D$ is a product of two primes $p$ and $q$, and from now on we assume that $D=p q$. Note however that dealing with the general case should not present conceptual difficulties.

In our case $D=p q$ it follows from the work of Ribet ([24], Proposition 4.4 and 4.7) that the set $\mathcal{V}$ of vertices of $\mathcal{G}$ consists of two copies $\mathcal{V}_{1}$ and $\mathcal{V}_{2}$ of the set of isomorphism classes of pairs $\left(E, C_{N}\right)$ where $E$ is a supersingular elliptic curve over $\overline{\mathbb{F}}_{q}$ and $C_{N} \subset E$ is a cyclic subgroup of order $N$ (notice the switch between $p$ and $q$ ). The set $\mathcal{E}$ of edges of the graph consists of $p$ isogenies between such objects. In other words the elements of $\mathcal{E}$ correspond to the isomorphism classes of triples $\left(E, C_{N}, C_{p}\right)$ where $C_{p}$ is a cyclic subgroup of order $p$, with source $\left(E, C_{N}\right)$ in $\mathcal{V}_{1}$ and target $\left(E / C_{p}, C_{N}+C_{p} / C_{p}\right)$ in $\mathcal{V}_{2}$. Note that this fixes an orientation on $\mathcal{G}$. The length of an edge corresponding to $\left(E, C_{N}, C_{p}\right)$ is $\operatorname{card}\left(\operatorname{Aut}\left(E, C_{N}, C_{p}\right) / \pm 1\right)$.

For each $d$ dividing $D N$ such that $\operatorname{gcd}(d, D N / d)=1$, there is a distinguished involution $w_{d}$ on the curve $X_{0}^{D}(N)_{\mathbb{Z}_{p}}$, a so-called Atkin-Lehner involution. The involution $w_{q}$ acts on $\mathcal{G}$ by sending $\left(E, C_{N}, C_{p}\right)$ to $\operatorname{Frob}_{q}\left(E, C_{N}, C_{p}\right)$ so if $C_{q}$ is the kernel in $E$ of Frob ${ }_{q}$ then $\operatorname{Frob}_{q}\left(E, C_{N}, C_{p}\right)=\left(E / C_{q}, C_{N}+C_{q} / C_{q}, C_{p}+C_{q} / C_{q}\right)$. Note that $w_{q}$ preserves the bipartition of $\mathcal{G}$. The involution $w_{p}$ maps an edge $\left(E, C_{N}, C_{p}\right)$ to $-\left(E / C_{p}, C_{N}+C_{p} / C_{p}, E[p] / C_{p}\right)$, therefore $w_{p}$ switches the bipartition of $\mathcal{G}$. Any quotient $X^{+}:=X / w_{d}$ is also an admissible curve and $\mathcal{G}^{+}:=\mathcal{G}\left(X_{\mathbb{F}_{p}} / w_{d}\right)=\mathcal{G}\left(X_{\mathbb{F}_{p}}\right) / w_{d}$ (taking into account the ramification in the length of the edges).

Let $J:=\operatorname{Jac}(X)$ and $J^{+}:=\operatorname{Jac}\left(X^{+}\right)$be the Néron models over $\operatorname{Spec}\left(\mathbb{Z}_{p}\right)$ of the jacobians of $X_{\mathbb{Q}_{p}}$ and $X_{\mathbb{Q}_{p}}^{+}$respectively. It follows from the previous section that the neutral components $J_{\mathbb{F}_{p}}^{0}$ and $J_{\mathbb{F}_{p}}^{+0}$ of $J_{\mathbb{F}_{p}}$ and $J_{\mathbb{F}_{p}}^{+}$are tori, and the character group of $J_{\mathbb{F}_{p}}^{0}$ (respectively, $J_{\mathbb{F}_{p}}^{+0}$ ) is isomorphic to $H_{1}(\mathcal{G}, \mathbb{Z})$ (respectively, $H_{1}\left(\mathcal{G}^{+}, \mathbb{Z}\right)$ ).

Let $\mathbb{T}$ be the Hecke algebra of $\Gamma_{0}(p q N)$. The results of Jacquet-Langlands imply that the elements of $\mathbb{T}$ define correspondences on the Shimura curve $X=X_{0}^{p q}(N)$. In order to formulate a version of Mestre-Oesterlé's graph method for Shimura curves, we need to understand the induced action of $\mathbb{T}$ on the special fiber of $J$. To achieve this we use a kind of "explicit twisted Jacquet-Langlands" which is at the heart of [24]. In this article Ribet compares the character group of $\operatorname{Jac}\left(X_{0}^{p q}(N)\right)_{\mathbb{F}_{p}}$ with a subgroup of the character group of $\operatorname{Jac}\left(X_{0}(p q N)\right)_{\mathbb{F}_{q}}$. To explain this result we introduce a few new notations. Let $\mathcal{X}$ be the group of degree 0 divisors on the set of supersingular points in $X_{0}(N q)\left(\mathbb{F}_{q^{2}}\right)$ and let $\mathcal{L}$ be the group defined in the analogous way for $X_{0}(N p q)$. The two natural degeneracy maps $\alpha$ and $\beta$ from $X_{0}(N p q)$ to $X_{0}(N q)$ induce maps $\alpha_{*}$ and $\beta_{*}$ from $\mathcal{L}$ to $\mathcal{X}$. Call $Y$ the kernel of the morphism $\left(\alpha_{*} \oplus \beta_{*}\right): \mathcal{L} \rightarrow \mathcal{X} \oplus \mathcal{X}$. Ribet shows that $\mathbb{T}$ restricts to an action on $Y$ which cuts-out the $p q$-new quotient of $\mathbb{T}([24]$, Theorem 3.20). Then he proves the following crucial result: 
Theorem 3.1 (Ribet) There is a $\mathbb{T}$-isomorphism (which is compatible with the monodromy pairings) between $Y$ and the character group of $\operatorname{Jac}\left(X_{0}^{p q}(N)\right)_{\mathbb{F}_{p}}^{0}$.

Proof. This is [24], Theorem 4.1.

Note that the fact that $Y$ is the character group of the jacobian of a Shimura curve already followed implicitly from the description of the graph given above.

\subsection{Lemmata on component groups.}

We close this section by proving technical lemmas on the asymptotic shape and the order of components of a Shimura curve $X^{p q}$ (or its quotient $X^{p q} / w_{q}$ ) embedded in its jacobian. We fix some more notations. Let $S=\left\{C_{0}, \ldots, C_{g}\right\}$ be the set of supersingular $j$-invariants in characteristic $q$. Write the bipartite graph $\mathcal{G}\left(X_{\mathbb{F}_{p}}^{p q}\right)$ as a union $\mathcal{V}_{1} \cup \mathcal{V}_{2}$. According to Ribet's description of the previous section, one may enumerate the vertices of $\mathcal{V}_{r}$ as $C_{r, 0}, \ldots, C_{r, g}$ for $r=1,2$. Each $C_{s} \in S$ corresponds to an elliptic curve $E_{s}$, and we set $w\left(C_{s}\right):=\operatorname{card}\left(\operatorname{Aut}\left(E_{s}\right) /( \pm 1)\right)$. One knows that two $w\left(C_{i}\right)$ at most are different from 1, in which case they are equal to 2 or 3 . Recall that the Eisenstein vector for $X_{0}(q)_{\overline{\mathbb{F}}_{q}}$ is Eis $:=\left(w\left(C_{0}\right)^{-1}, w\left(C_{1}\right)^{-1}, \ldots, w\left(C_{g}\right)^{-1}\right)^{t} \in \frac{1}{12} \mathbb{Z}^{S}$. For $v=\left(v_{i}\right)_{i \in S} \in \mathbb{C}^{S}$ we define the weight $w(v)$ as $\sum_{i \in S} v_{i}$. The weight $w($ Eis $)=\sum w\left(C_{r}\right)^{-1}$ is roughly $g\left(X_{0}(q)\right) \simeq q / 12$.

Lemma 3.3 Fix a prime $q>3$. As the prime $p$ tends to infinity, the edges of $\mathcal{G}\left(X_{\mathbb{F}_{p}}^{p q}\right)$ are equidistributed in the following sense. If $C_{1, j}$ and $C_{2, i}$ are elements of $\mathcal{V}_{1}$ and $\mathcal{V}_{2}$ respectively, the number of edges from $C_{1, j}$ to $C_{2, i}$ is

$$
\frac{(p+1)}{w(\text { Eis })} \times \frac{1}{w\left(C_{1, j}\right) w\left(C_{2, i}\right)}+O_{q}(\sqrt{p}) .
$$

Proof. We first deal with the generic case: when $w\left(C_{1, j}\right)=1$. Considering the transpose $T_{p} \in M_{g+1}(\mathbb{Z})$ of the $p^{\text {th }}$-Brandt matrix for $\Gamma_{0}(q)$ with respect to the basis associated to $S$, one sees that the number of edges from $C_{1, j}$ to $C_{2, j}$ is the $i^{\text {th }}$ coefficient of the $j^{\text {th }}$ column vector of $T_{p}$ (cf. for instance Proposition 4.4 of [13]). Write $C_{j}=(0, \ldots, 0,1,0, \ldots, 0)^{t}$ in $\mathbb{Z}^{S}$, where the coefficient 1 occurs at the $j^{\text {th }}$ place. Decompose $C_{j}$ as $(1 / w($ Eis $))$ Eis $+c_{j}$, with $c_{j}$ a vector of weight 0 . Now the lemma follows from the fact that $T_{p}($ Eis $)=(p+1)$ Eis, together with Weil's bounds on cuspidal eigenvalues (i.e. if $a_{p}$ is such an eigenvalue, with $p$ prime to the level, then $\left|a_{p}\right| \leq 2 \sqrt{p}$, see e.g. [9], 5.0.1 and references therein).

When $w\left(C_{1, j}\right)=2$ or 3 , one uses the same arguments, plus the fact that, on a supersingular elliptic curve in characteristic $q$ corresponding to $C_{1, j}$, the $p$-isogenies which are conjugated by 
the exceptional automorphism $\zeta\left(=\zeta_{4}\right.$ or $\left.\zeta_{3}\right)$ are isomorphic. Identifying the set of $p$-isogenies with $\mathbb{P}^{1}\left(\mathbb{F}_{p}\right)$ one sees that, depending on the decomposition of $p$ in $\mathbb{Q}(\zeta)$, the exceptional isomorphism may fix zero, one or two of these isogenies; the others belong to orbits of length $w\left(C_{1, j}\right)$. However the quantitative form of the statement does not depend on these different cases.

Lemma 3.4 Assume that $p$ and $q$ verify the hypotheses of Theorem 1.1, case 2 with $p, q>3$. Let $C$ be a vertex of $\mathcal{G}\left(\left(X^{p q} / w_{q}\right)_{\mathbb{F}_{p}}\right)$ associated to the $\operatorname{Gal}\left(\mathbb{F}_{q^{2}} / \mathbb{F}_{q}\right)$-orbit of a supersingular invariant $j$. The number of edges emanating from $C$ is:

1. $(p+1)$ if $j$ belongs to $\mathbb{F}_{q^{2}} \backslash \mathbb{F}_{q}$;

2. $(p+1) / 2$ if $j \in \mathbb{F}_{q}$ and $j \not \equiv 0,1728$;

3. $(p+3) / 4$ if $j \equiv 1728$;

4. $(p+3 \pm 2) / 6$ if $j \equiv 0$.

Setting $\varepsilon\left(C_{1, s}, C_{2, r}\right)=1$ if $C_{1, s}$ or $C_{2, r}$ belongs to $\mathbb{F}_{q^{2}} \backslash \mathbb{F}_{q}$, and $\varepsilon\left(C_{1, s}, C_{2, r}\right)=2$ if both lie in $\mathbb{F}_{q}$, the number $N_{C_{1, s}, C_{2, r}}$ of edges between two vertices $C_{1, s}$ and $C_{2, r}$ belonging to two different sets of the bipartition of $\mathcal{G}\left(\left(X^{p q} / w_{q}\right)_{\mathbb{F}_{p}}\right)$ is:

$$
N_{C_{1, s}, C_{2, r}}=\frac{p+1}{w(\text { Eis })} \times \frac{1}{\varepsilon\left(C_{1, s}, C_{2, r}\right) w\left(C_{1, s}\right) w\left(C_{2, r}\right)}+O_{q}(\sqrt{p}) .
$$

Proof. The first part of the lemma follows form Ribet's description of the graph (sketched before Theorem 3.1). Indeed, let $\mathcal{C}$ be a vertex of $\mathcal{G}\left(X_{\mathbb{F}_{p}}^{p q}\right)$ associated to a supersingular curve $E$ with invariant $j$. We recall that the involution $w_{q}$ acts as $\operatorname{Frob}_{q}$ on $\mathcal{G}\left(X_{\mathbb{F}_{p}}^{p q}\right)$. If $j$ belongs to $\mathbb{F}_{q^{2}} \backslash \mathbb{F}_{q}$, there are $p+1$ edges emanating from $\mathcal{C}$ in $\mathcal{G}\left(X_{\mathbb{F}_{p}}^{p q}\right)$, and as $w_{q}(\mathcal{C}) \neq \mathcal{C}$, the same is true for its image $C$ in $\mathcal{G}\left(\left(X^{p q} / w_{q}\right)_{\mathbb{F}_{p}}\right)$, so case 1 of the lemma follows. Suppose that $j$ belongs to $\mathbb{F}_{q}$, in which case $\mathcal{C}$ is fixed by $w_{q}$. The hypotheses on $p$ and $q$ insure that that no edge of $\mathcal{G}\left(X_{\mathbb{F}_{p}}^{p q}\right)$ is fixed under the action of $w_{q}$ (see [27], proof of Theorem 3.1 and [21], p. 204 et seq.). This allows to conclude in case 2 of Lemma 3.4. If $j \equiv 1728$ (respectively, $j \equiv 0$ ), one must take into account the fact that $E$ has an extra automorphism of order 4 (respect. of order 6 ) as in the proof of Lemma 3.3. As we assume that $p \equiv 1 \bmod 4$, this shows that there are only $(p+3) / 2$ (respect. $(p+3 \pm 2) / 3)$ edges emanating from $\mathcal{C}$, and half this number from $C$. This proves the cases 3 and 4 of Lemma 3.4. The assertion about $N_{C_{1, s}, C_{2, r}}$ is clear from Lemma 3.3.

To prove the next lemma we need an explicit description of component groups. To achieve that purpose we recall the method of the proof of [11], Proposition 9.2. See also [10], section 1. 
Let $\mathcal{G}$ be a graph with the set of vertices and edges $S_{0}$ and $S_{1}$ respectively, and write $\mathbb{Z}^{S_{0}}[+]$ for the kernel of the "weight" map $f \mapsto \sum_{v \in S_{0}} f(v)$ from $\mathbb{Z}^{S_{0}}$ to $\mathbb{Z}$. Raynaud's results show that the component group $\Phi$ of the jacobian of an admissible curve with the graph $\mathcal{G}$ is the cokernel of the map $\iota: \mathbb{Z}^{S_{0}} \rightarrow \mathbb{Z}^{S_{0}}[+]$ given by the intersection matrix of $\mathcal{G}$. More precisely, if $\Delta: \mathbb{Z} \rightarrow \mathbb{Z}^{S_{0}}$ is the diagonal map, one has the exact sequence:

$$
0 \rightarrow \mathbb{Z} \stackrel{\Delta}{\rightarrow} \mathbb{Z}^{S_{0}} \stackrel{\iota}{\rightarrow} \mathbb{Z}^{S_{0}}[+] \rightarrow \Phi \rightarrow 0
$$

(see e.g. [11], loc. cit.). Now having fixed an orientation on $\mathcal{G}$, let $s$ and $t$ be the obvious "source" and "target" maps from $S_{1}$ to $S_{0}$. Define $t^{*}: \mathbb{Z}^{S_{0}} \rightarrow \mathbb{Z}^{S_{1}}$ by $\left(t^{*} f\right)(e)=f(t(e))$ and $t_{*}: \mathbb{Z}^{S_{1}} \rightarrow \mathbb{Z}^{S_{0}}$ by $\left(t_{*} f\right)(v)=\sum_{t(e)=v} f(e)$. Define similarly $s^{*}$ and $s_{*}$ and set $d^{*}:=t^{*}-s^{*}$ and $d_{*}:=t_{*}-s_{*}$. One readily checks that the map $\iota$ can be factorized as $\iota=-d_{*} d^{*}$ (cf. for instance [8], section 1.2, proof of Theorem 1.2.3).

The basic problem we discuss in the next lemma is the following: given an element in $\mathbb{Z}^{S_{0}}[+]$ of the form $(p+1)\left(C_{1}-C_{2}\right)$, is it in the image of $\iota$, or not? Recall that for $X$ an admissible curve, $\tilde{X}$ denotes the regular curve obtained from $X$ by blowing-up.

Lemma 3.5 Fix a prime $q$ such that $g\left(X_{0}(q)\right) \geq 5$ and suppose that $p \equiv 5 \bmod 12$ and $q \equiv 3$ mod 4. For $p$ large enough (compared to $q$ ), if a non-trivial difference between two irreducible components of $\left(\widehat{X^{p q} / w_{q}}\right)_{\mathbb{F}_{p}}$ is killed by $(p+1)$ in the component group of $\operatorname{Jac}\left(X^{p q} / w_{q}\right)_{\mathbb{F}_{p}}$, then these two components belong to the same set $\mathcal{V}_{1}$ or $\mathcal{V}_{2}$ of the bipartition of the set of components.

In particular, if $\mathcal{J}$ denotes the exceptional component arising from multiplication by $\zeta_{4}$ and $J$ is any other component, then $(\mathcal{J}-J)$ is not killed by $p+1$.

Proof. We consider the graph $\mathcal{G}^{+}:=\mathcal{G}\left(\left(\widetilde{X^{p q} / w_{q}}\right)_{\mathbb{F}_{p}}\right)$, whose sets of vertices and edges are still denoted by $S_{0}$ and $S_{1}$ respectively. As we assume that $p \equiv 5 \bmod 12$ and $q \equiv 3 \bmod 4$, we know that there is exactly one exceptional component $\mathcal{J}$ arising from multiplication by $\zeta_{4}$, and no exceptional component arising from multiplication by $\zeta_{3}$ (see [21], pp. 205 et seq., or the discussion preceding Theorem 4.1 and the proof of Lemma 5.1 of the present paper. Note that this does not prevent a vertex from having "multiplication by $\zeta_{3}$ ", i.e. from corresponding to a supersingular invariant $j \equiv 0 \bmod q$ ). Let $w:=w($ Eis) be the weight of the Eisenstein vector for $X_{0}(q)_{\mathbb{F}_{q}}$ as in the Lemma 3.3. The hypothesis $g\left(X_{0}(q)\right) \geq 5$ implies $w>4$. For $C \in S$ write $\varepsilon(C)=2$ if $C \in \mathbb{F}_{q}$, and $\varepsilon(C)=1$ otherwise. Recall that the set of vertices of $\mathcal{G}^{+}$other than $\mathcal{J}$ is $\mathcal{V}_{1} \cup \mathcal{V}_{2}$. Call $J_{1}$ and $J_{2}$ the components surrounding $\mathcal{J}$. One has $w\left(J_{1}\right)=w\left(J_{2}\right)=2$. There are exactly two edges emanating from $\mathcal{J}$, one towards $J_{1}$ and one towards $J_{2}$. Let $J$ be a component (possibly $J_{1}$ ) belonging to the set $\mathcal{V}_{1}$, say. We divide the set of vertices of $\mathcal{G}^{+}$other than $\mathcal{J}, J, J_{1}$, $J_{2}$ into the subsets $\left\{j_{i, 1}\right\}$ and $\left\{j_{i, 2}\right\}$ of $\mathcal{V}_{1}$ and $\mathcal{V}_{2}$ respectively. By Lemma 3.4 if $\left(C_{1}, C_{2}\right)$ belongs 
to $\mathcal{V}_{1} \times \mathcal{V}_{2}$ then $N_{C_{1}, C_{2}}>0$ for $p \gg q$. We suppose this to be the case in what follows. (At this point, it may help the reader to make a rough sketch of what $\mathcal{G}^{+}$looks like.) The symbol $O_{q}(\sqrt{p})$ below will denote a priori different functions, which depend on the vertices of our graph, but as $q$ is fixed the set of vertices is fixed too, therefore those functions can be bounded by a single one. To prove Lemma 3.5 we have several kinds of generic configurations to consider for our differences $\left(C_{1}-C_{2}\right)$, relative to the above data. Using the previous lemmas the computations are elementary but lengthy so we treat in detail only the cases of the last statement of the lemma and we leave other cases to the reader. (Note that in the computations below, we only need $w>3$, but the assumption $w>4$ in our statement is necessary for the cases we do not write down here). From now on we assume that there is a $v \in \mathbb{Z}^{S_{0}}$ such that $d_{*} d^{*}(v)=(p+1)(J-\mathcal{J})=: i$. The discussion before the lemma shows that we need to obtain a contradiction for $p \gg q$.

We point out that our problem has a simple electrodynamical interpretation. Indeed, consider $\mathcal{G}^{+}$as an electric circuit in which every edge has a resistance of one (ohm, say). The element $i \in \mathbb{Z}^{S_{0}}[+]$ may be seen as a vector of current: at each node $C \in S_{0}$, a current of $i(C)$ ampères enters the circuit. If $v \in \mathbb{Q}^{S_{0}}$ satisfies $d_{*} d^{*}(v)=i$ then $v$ can be interpreted as a distribution of potential in volts at each node, corresponding to the current $i$ on $\mathcal{G}^{+}$. From this point of view our problem boils down to proving that no integer valued distribution of potential on the nodes allows a current of $p+1$ ampères to enter the circuit at $J$ and leave it at $\mathcal{J}$. This electrodynamical interpretation is a common practice among graph theorists (see for instance [4], II.1). It is also used in [11], Proposition 9.2.

Sublemma 3.5.1 ("law (K)"). With the above assumptions and notations, at each vertex $C$ in $S_{0}$ one has $\sum_{D \mapsto C}(v(C)-v(D))=i(C)$, where " $\sum_{D \mapsto C}$ " means the sum over the neighbors of $C$ in $\mathcal{G}^{+}$.

Proof of 3.5.1. Clear from the fact that $i=d_{*} d^{*}(v)$.

(Note that, from the electrodynamical point of view, this is just the conjunction of Ohm's law and Kirchhof's law (see [4], loc. cit.). Indeed, Ohm's law says that if a current $c$ passes through a wire of resistance $r$ between two nodes $A$ and $B$ with a difference in potential equal to $u$, then $u=$ r.c. On the other hand, Kirchhoff's law says that the sum of currents passing through a node is zero. In our case each wire in $S_{1}$ has resistance $r=1$ and $d^{*}(v) \in \mathbb{Z}^{S_{1}}$ is the distribution of current through the wires of $\mathcal{G}^{+}$.)

Sublemma 3.5.2 With the above hypotheses and notations, one has $v(\mathcal{J})<v(C)<v(J)$ for every vertex $C$ different from $J$ and $\mathcal{J}$. 
Proof of 3.5.2. Let $C_{0}$ be such that $v\left(C_{0}\right)$ is maximal among the $v(C)$ with $C \neq J$ and suppose that $v\left(C_{0}\right) \geq v(J)$. By connexity of $\mathcal{G}^{+}$one may assume that the vertex $C_{0}$ has a neighbor $D$ such that $v(D)<v\left(C_{0}\right)$ (otherwise $v$ would be constant on $\mathcal{G}^{+}$, a contradiction with the exact sequence $(R)$ before Lemma 3.5). Therefore $\sum_{D \mapsto C_{0}}\left(v\left(C_{0}\right)-v(D)\right)>0$, which is a contradiction with (3.5.1) as $i\left(C_{0}\right) \leq 0$. The other inequality of the sublemma is obtained by symmetry.

(Note that from the electrodynamical point of view, the sublemma just says that the distribution of potential must decrease between the nodes $J$ (where the current enters) and $\mathcal{J}$ (where it comes out)).

We finally turn to the proof of the Lemma 3.5 itself, examining the different configurations that may occur for $J$ and $\mathcal{J}$. As we feel that the electrodynamical language gives slightly shorter and clearer proofs, we will use it freely. For readers who prefer more formal proofs however, we treat our first case below in both abstract and physical languages. It is our hope that this will clarify our method for the rest of the computations.

Let $\mathcal{J}_{2} \in\left\{J_{2}\right\} \cup \mathcal{V}_{2}$ be such that $v\left(\mathcal{J}_{2}\right)=\max \left(v\left(j_{i, 2}\right), v\left(J_{2}\right)\right)$. Lemma 3.4 insures that there are $(p+1) / \varepsilon(J) w(J)$ edges leaving $J$. Recall that we assume that for each pair of vertices $\left(C_{1}, C_{2}\right)$ in $\mathcal{V}_{1} \times \mathcal{V}_{2}$ the number $N_{C_{1}, C_{2}}$ of edges between $C_{1}$ and $C_{2}$ is nonzero. By (3.5.2) one has $v(J)>v\left(J_{2}\right), v(J)>v\left(j_{i, 2}\right)$ for all $j_{i, 2}$ in $\mathcal{V}_{2}$, and by (3.5.1), $(p+1)=N_{J, J_{2}}\left(v(J)-v\left(J_{2}\right)\right)+\sum_{j_{i, 2}} N_{J, j_{i, 2}}\left(v(J)-v\left(j_{i, 2}\right)\right) \geq\left(v(J)-v\left(\mathcal{J}_{2}\right)\right)\left(N_{J, J_{2}}+\sum_{j_{i, 2}} N_{J, j_{i, 2}}\right)$ $=\left(v(J)-v\left(\mathcal{J}_{2}\right)\right)(p+1) / \varepsilon(J) w(J)$, therefore $v(J)-v\left(\mathcal{J}_{2}\right) \leq \varepsilon(J) w(J)$.

Case 1: Assume that $J \neq J_{1}$.

1a: Suppose that $v$ is constant on $\left\{J_{2}\right\} \cup \mathcal{V}_{2}$.

(Abstract language.) One has $v(J)-v\left(\mathcal{J}_{2}\right)=v(J)-v\left(J_{2}\right)=\varepsilon(J) w(J)$ by the preceding computation. Applying the law $(K)$ (3.5.1) at each $j_{i, 1}$ in $\mathcal{V}_{1}$ shows that the $v\left(j_{i, 1}\right)$ are constant, equal to $v\left(J_{2}\right)$. Applying (3.5.1) again at $J_{1}$, then at each $j_{i, 2}$, then at $J$, gives that $v\left(J_{1}\right)-v(\mathcal{J})=\left(v\left(J_{2}\right)-v\left(J_{1}\right)\right) N_{J_{2}, J_{1}}+\sum_{j_{i, 2}} N_{j_{i, 2}, J_{1}}\left(v\left(j_{i, 2}\right)-v\left(J_{1}\right)\right) \geq \sum_{j_{i, 2}} N_{j_{i, 2}, J_{1}}\left(v\left(j_{i, 2}\right)-v\left(J_{1}\right)\right)$ $\geq \sum_{j_{i, 2}} N_{J, j_{i, 2}}\left(v(J)-v\left(j_{i, 2}\right)\right)=(p+1)-\left(v(J)-v\left(J_{2}\right)\right) N_{J, J_{2}}=(p+1)\left(1-\frac{\varepsilon(J)}{2 \varepsilon\left(J, J_{2}\right) w}\right)+O_{q}(\sqrt{p})$ by Lemma 3.4. Now $v\left(J_{2}\right)-v(\mathcal{J})>v\left(J_{1}\right)-v(\mathcal{J})$ so (3.5.1) applied at $J_{2}$ gives $(p+1)\left(1-\frac{\varepsilon(J)}{2 \varepsilon\left(J, J_{2}\right) w}\right)+O_{q}(\sqrt{p}) \leq\left(v(J)-v\left(J_{2}\right)\right) N_{J_{2}, J}=(p+1) \frac{\varepsilon(J)}{2 \varepsilon\left(J, J_{2}\right) w}+O_{q}(\sqrt{p})$. This implies $w \leq 1$ for $p \gg q$.

(Electrodynamical language.) By the law $(K)$ at each $j_{i, 1}$ in $\mathcal{V}_{1}$, one sees that the $v\left(j_{i, 1}\right) \mathrm{s}$ are constant and equal to $v\left(J_{2}\right)$. Therefore all the current arriving from $J$ to the $j_{i, 2} \mathrm{~s}$ goes to $J_{1}$. It then goes to $\mathcal{J}$, and this total current is at least $(p+1)\left(1-\frac{\varepsilon(J)}{2 \varepsilon\left(J, J_{2}\right) w}\right)+O_{q}(\sqrt{p})$ by Lemma 3.4. This means that $v\left(J_{1}\right)-v(\mathcal{J})$ is larger than this latter quantity, and the same is a fortiori true of 
$v\left(J_{2}\right)-v(\mathcal{J})$. But the current that $J_{2}$ receives from $J$ is only $(p+1) \frac{\varepsilon(J)}{2 \varepsilon\left(J, J_{2}\right) w}+O_{q}(\sqrt{p})$, and this implies $w \leq 1$ for $p \gg q$.

1.b: Suppose that $v$ is not constant on $\left\{J_{2}\right\} \cup \mathcal{V}_{2}$.

We first note that we have $\varepsilon(J)=2$, because if $J$ is quadratic over $\mathbb{F}_{q}$ there are $(p+1)$ edges emanating from $J$, therefore (3.5.1) implies that $v$ is constant and equal to $v(J)-1$ on $\left\{J_{2}\right\} \cup \mathcal{V}_{2}$. As there is a $j_{2}$ in $\left\{J_{2}\right\} \cup \mathcal{V}_{2}$ such that $v\left(j_{2}\right)<v\left(\mathcal{J}_{2}\right)$ one sees that $v\left(\mathcal{J}_{2}\right)>v\left(J_{1}\right)$ (otherwise we get a contradiction with the fact that $J_{1}$ has no current to offer to $\left.j_{2}\right)$, and similarly $v\left(\mathcal{J}_{2}\right)>v\left(j_{i, 1}\right)$ for all $j_{i, 1}$ in $\mathcal{V}_{1}$. Therefore the node $\mathcal{J}_{2}$ has to deal at least $\frac{p+1}{\varepsilon\left(\mathcal{J}_{2}\right) w\left(\mathcal{J}_{2}\right)}\left(1-\frac{1}{w \cdot w(J)}\right)+O_{q}(\sqrt{p})$ ampères, whereas it receives (from $J$ ) strictly less than $\frac{(p+1)}{\varepsilon\left(\mathcal{J}_{2}\right) w\left(\mathcal{J}_{2}\right) w(J) w}(\varepsilon(J) w(J))+O_{q}(\sqrt{p})$. This gives $w \leq \varepsilon(J)+1 / w(J) \leq 3$ for $p \gg q$.

Case 2. Assume that $J=J_{1}$. As in the case 1.b, the node $\mathcal{J}_{2}$ has to deal at least $\frac{p+1}{\varepsilon\left(\mathcal{J}_{2}\right) w\left(\mathcal{J}_{2}\right)}\left(1-\frac{1}{2 w}\right)+O_{q}(\sqrt{p})$ ampères, whereas it receives from $J_{1}$ less than $\frac{(p+1)}{2 \varepsilon\left(\mathcal{J}_{2}\right) w\left(\mathcal{J}_{2}\right) w}\left(v\left(J_{1}\right)-v\left(\mathcal{J}_{2}\right)\right)+O_{q}(\sqrt{p}) \leq \frac{2(p+1)}{\varepsilon\left(\mathcal{J}_{2}\right) w\left(\mathcal{J}_{2}\right) w}+O_{q}(\sqrt{p})$. This gives $w \leq 5 / 2$ for $p \gg q$.

This completes the examination of the cases of differences of components of shape $\mathcal{J}-J$, which will be the only ones of interest to us in the following of the paper. The other cases of the lemma are similarly elementary and lengthy to treat.

Remark 3.6 The above makes it clear that, if $p$ is large enough relatively to $q$, the graphs $\mathcal{G}$ or $\mathcal{G}^{+}$are "non-disconnecting". It follows that the maps from $X_{\mathbb{Z}_{p}}^{p q, \mathrm{sm}}$ and $\left(X^{p q} / w_{q}\right)_{\mathbb{Z}_{p}}^{\text {sm }}$ to their jacobian over the same basis are closed immersions, by Proposition 9.5 of [11]. We will use this remark before the Theorem 5.3.

\section{Shimura curves and results of Gross, Zhang and Wald- spurger.}

In this section we show how the results of Gross, Zhang and Waldspurger on the special values of automorphic $L$-functions describe the winding quotient of the jacobian of our Shimura curves, generalizing [22], Proposition 4.2.

Let $G_{\mathbb{Q}}$ denote the absolute Galois group of $\mathbb{Q}$ and $S_{2}^{\text {new }}\left(\Gamma_{0}(N)\right)_{\overline{\mathbb{Q}}}$ the set of newforms over $\overline{\mathbb{Q}}$ of weight 2 for $\Gamma_{0}(N)$. For any integer $N$, the theory of Shimura associates to the decomposition into $G_{\mathbb{Q}}$-orbits of $S_{2}^{\text {new }}\left(\Gamma_{0}(N)\right)_{\overline{\mathbb{Q}}}$ a similar decomposition over $\mathbb{Q}$, up to isogeny, of $J_{0}(N)$ into a product $\prod_{G_{\mathbb{Q}}-\text { orbits }} A_{f}$. According to Shimura, Carayol et al., one has

$$
L\left(J_{0}(N), s\right)=* \prod_{G_{\mathbb{Q}}-\text { orbits }} L\left(A_{f}, s\right)=* \prod_{G_{\mathbb{Q}}-\text { orbits }} \prod_{\left(f^{\sigma} \in G_{\mathbb{Q}} \cdot f\right)} L\left(f^{\sigma}, s\right)
$$


(where the $*$ s denote non-zero constants). The Hecke algebra $\mathbb{T}:=\mathbb{T}_{\Gamma_{0}(N)}$ is a free $\mathbb{Z}$-module of finite type. The above decomposition of $J_{0}(N)$ corresponds to idempotents in $\mathbb{T} \otimes \mathbb{Q}$ and there is a unique ideal $I_{e}$ of $\mathbb{T}$ such that $J_{0}(N) / I_{e} J_{0}(N)$ is isogenous to $\prod_{L\left(A_{f}, 1\right) \neq 0} A_{f}$ and $I_{e}$ is saturated (i.e. equal to the inverse image of $I_{e} \otimes \mathbb{Q}$ under the map $\mathbb{T} \rightarrow \mathbb{T} \otimes \mathbb{Q}$ ). This quotient abelian variety is called the winding quotient and we denote it by $J_{e}(N)$. A milestone theorem of Kolyvagin-Logachev (see [19]), generalized by Kato in [18], implies that $J_{e}(N)(\mathbb{Q})$ is finite (this is part of a special case of the conjecture of Birch and Swinnerton-Dyer). The theory of Jacquet-Langlands establishes a correspondence between automorphic forms for $\mathrm{GL}_{2}$ on the one hand, and automorphic forms for the multiplicative group of nonsplit quaternion algebras, on the other hand (see e.g. [16]). This implies that one can also make the above construction for any (quotient of) a general Shimura curve instead of the classical $X_{0}(N)$.

We want to develop a graph method on our Shimura curves in a similar way to [22]. The $\mathbb{Z}$-modules $\mathcal{L}$ and $Y$ of the end of section 3.1 can be interpreted as character groups or, as we are working with tori, as spaces of differential 1-forms on the corresponding geometric objects. The use of special values formulae for suitable Rankin-Selberg $L$-functions allows us to describe in these spaces the subspaces of differentials pertaining to the winding quotient. To be more precise we need to fix some notations and recall some results from Gross' theory (a neat introduction to this circle of ideas can be found in [32]). If $M$ is a $\mathbb{Z}$-module, define $\hat{M}:=M \otimes \hat{\mathbb{Z}}$. Let $B$ be the quaternion algebra over $\mathbb{Q}$ which is ramified precisely at $q$ and $\infty$. Choose a Eichler order $R$ of level $p$ in $B$, and let $\left\{R_{1}:=R, \ldots, R_{n}\right\}$ be a set of Eichler orders in $B$ corresponding to representatives for $\hat{R}^{*} \backslash \hat{B}^{*} / B^{*}$ : to a double coset $g:=\left(g_{2}, g_{3}, \ldots, g_{l}, \ldots\right)$ we associate the $B^{*}$-conjugation class of the Eichler order $B \cap g^{-1} \hat{R} g$. Recall that $\hat{R}^{*} \backslash \hat{B}^{*} / B^{*}$ is in one-to-one correspondence with the set $\mathcal{S}$ of singular points of $X_{0}(p q)_{\mathbb{F}_{q}}$ (see [24], 3.3 and 3.4). The order $R_{i}$ associated to a point $x=\left(E, C_{p}\right)$ is such that $R_{i} \simeq \operatorname{End}_{\overline{\mathbb{F}}_{q}}\left(E, C_{p}\right)$.

If $L$ is a quadratic number field, it embeds in $B$ if and only if its localization at ramification primes for $B$ is a field, i.e. $L$ is a quadratic imaginary field in which $q$ is inert or ramified. Then, for an order $\mathcal{O}$ of $L$, a morphism of algebras $\sigma: L \hookrightarrow B$, and a Eichler order $\mathcal{R}$ as above, the pair $(\sigma, \mathcal{R})$ is said to be an optimal embedding of $\mathcal{O}$ in $\mathcal{R}$ if $\sigma(L) \cap \mathcal{R}=\sigma(\mathcal{O})$. Such an embedding exists if and only if $p$ splits or ramifies in $\mathcal{O}$. If $-D$ is a negative integer, let $h(-D)$ be the class number of the quadratic order $\mathcal{O}_{-D}$ with discriminant $-D$ (if it exists), and let $h_{i}(-D)$ be the number of optimal embeddings of $\mathcal{O}_{-D}$ in $R_{i}$ modulo conjugation by $R_{i}^{*}$. We define in $\mathbb{Z}^{\mathcal{S}}$ the element:

$$
e_{D}:=\sum_{i=1}^{n} \frac{h_{i}(-D)}{\left|\operatorname{Aut}\left(R_{i}^{*} / \pm 1\right)\right|}\left[R_{i}\right] .
$$

We call those elements Gross vectors.

Now let $f$ be a newform of weight 2 for $\Gamma_{0}(p q)$. If $-D$ is a quadratic imaginary discriminant 
as above, we write $-D=-d \cdot c^{2}$, where $-d$ is a fundamental discriminant and $c$ a conductor. Let $\varepsilon_{D}$ be the non-trivial quadratic Dirichlet character associated to $\mathbb{Q}(\sqrt{-D})$, and $f \otimes \varepsilon_{D}$ the twist of $f$ by $\varepsilon_{D}$. One knows that the natural action of the Hecke operators as correspondences endows $\mathcal{L}$ with a structure of $\mathbb{T}$-module (the Brandt Hecke module), such that $\mathcal{L} \otimes \mathbb{Q}$ is a free $\mathbb{T} \otimes \mathbb{Q}$-module of rank 1 (see [13], section 5, for the case of prime level, and for instance [12] for more general results). Let $(\mathcal{L} \otimes \overline{\mathbb{Q}})^{f}$ be the $\mathbb{T}_{\overline{\mathbb{Q}}}$-eigenspace in $\mathcal{L} \otimes \overline{\mathbb{Q}}$ associated to $f$, and let $e_{f, D}$ be the component of $e_{D}$ on $(\mathcal{L} \otimes \overline{\mathbb{Q}})^{f}$.

Theorem 4.1 (Gross, Zhang, Waldspurger) With notations as above, the number $L(f, 1) L\left(f \otimes \varepsilon_{D}, 1\right)$ is non-zero if and only if $e_{f, D}$ is non-zero.

Proof. This follows from a result of Waldspurger, as explained, in a slightly different language, in section 1.4 of [7]. Under the assumption that $(p q, D)=1$ and $(c, p q D)=1$, see also [32], Theorem 6.4. Note that, when $f$ has prime level, an exact formula expressing $L(f, 1) L\left(f \otimes \varepsilon_{D}, 1\right)$ in terms of $e_{f, D}$ was proved by Gross in his seminal work [13], Corollary 11.6. This was generalized in [33], Theorem 1.3.2, or [34], Theorem 7.1. It was this formula that we used in [22]. But even the newest versions of this exact Gross-Zhang formula require mildly restrictive hypotheses. In the rest of this work however, as in the arithmetic applications of [7], one only needs the nonvanishing statement of Theorem 4.1.

We now come back to the case of modular curves. It follows from [24], Proposition 3.1, that the group $\mathcal{L}$ of section 3.1 is isomorphic as a $\mathbb{T}$-module to the character group of the maximal subtorus of $J_{0}(p q)_{\mathbb{F}_{q}}$.

Proposition 4.2 Let $\mathbb{E}$ be the $\mathbb{Q}$-vector subspace of $\mathcal{L} \otimes \mathbb{Q}$ spanned by the orthogonal projections of Gross vectors (relative to the monodromy pairing $\langle\cdot, \cdot\rangle$, cf. section 2). Then $\mathbb{E}=\mathcal{L}\left[I_{e}\right] \otimes \mathbb{Q}$.

Proof. This is a straightforward variant of [22], Proposition 4.2.

We obtain an analogous result in the case of Shimura curves.

Corollary 4.3 The subspace $\mathbb{E} \cap Y_{\mathbb{Q}}$ of $Y_{\mathbb{Q}}=Y \otimes \mathbb{Q}$ is equal to the character group of the winding quotient of $J_{0}^{p q-\text { new }}(p q)$, i.e. the character group of the winding quotient of $\operatorname{Jac}\left(X^{p q}\right)_{\mathbb{F}_{p}}^{0}$.

Proof. Combine Proposition 4.2 with Theorem 3.1.

The Corollary 4.3 says that homology classes of the closed paths in the graph of $X_{\mathbb{F}_{p}}^{p q}$ made of Gross vectors are characters of the winding quotient. This gives a concrete method for obtaining those characters, which is a crucial point of our strategy. 


\section{General method.}

We turn to our initial problem of proving the triviality of the rational points on Atkin-Lehner quotients of Shimura curves. Set as before $X=X^{p q} / w_{q}$. Suppose that there exists a point $P$ in $X(\mathbb{Q})$, and let $P_{0}:=w_{p}(P)$. Our aim is to prove that $P=P_{0}$, which implies that $P$ is a special (or CM) point (cf. e.g. the first chapter of [6] or [2], Remark 6.5) - or leads to a contradiction if there are no special points in $X(\mathbb{Q})$. Consider the following diagram of $\mathbb{Q}$-morphisms:

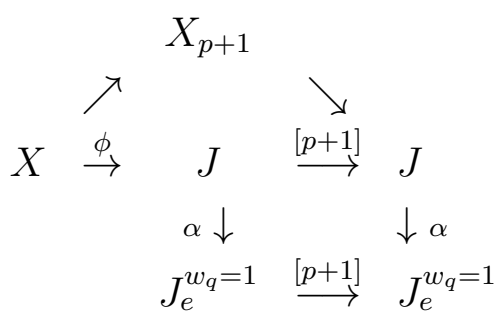

where $\phi(Q)=(Q)-\left(P_{0}\right), X_{p+1}=[p+1] \circ \phi(X)$ as at the end of section 2, $\alpha$ is the projection of $J=\operatorname{Jac}(X)$ to its winding quotient $J_{e}^{w_{q}=1}$ (isogenous to $J_{e} /\left(1-w_{q}\right) J_{e}$ for $J_{e}$ the winding quotient of $\left.\operatorname{Jac}\left(X^{p q}\right)\right)$. It is clear that everything commutes in this diagram. We define $\psi=[p+1] \circ \phi$ and $\Psi=\alpha \circ \psi$.

Let $\tilde{X}$ be the desingularization of $X$ over $\mathbb{Z}_{p}$ as in section 2 . As $\tilde{X}$ is regular, any point in $\tilde{X}\left(\mathbb{Z}_{p}\right)$ belongs to $\tilde{X}^{\text {sm }}\left(\mathbb{Z}_{p}\right)$. For such a $Q$ in $\tilde{X}\left(\mathbb{Z}_{p}\right)$, let $C_{Q}$ be the vertex of $\mathcal{G}(\tilde{X})$ corresponding to the specialization component of $Q$.

Lemma 5.1 Assume that $p$ and $q$ satisfy the hypotheses of Theorem 1.1, case 2. Then $\tilde{X}_{\mathbb{F}_{p}}$ has a unique $\mathbb{F}_{p}$-rational component which is necessarily $C_{P_{0}}$. This component intersects the other components in two points exactly. The embedding $\phi$ identifies $C_{P_{0}}^{\mathrm{sm}}$ with a one-dimensional subtorus $T$ of $J$ which is isomorphic to the non-trivial twist of $\mathbb{G}_{m}$ over $\mathbb{F}_{p}$ (cf. Proposition 2.1). In particular, for any $Q$ in $\tilde{X}\left(\mathbb{Z}_{p}\right)$, one has $C_{Q}=C_{w_{p}(Q)}=C_{P_{0}}$.

Proof. We use again the work of Ogg ([21]), Théorème of p. 206. In loc. cit. indeed, as we already noticed, it is explained that the vertices and the edges of $\mathcal{G}\left(X_{\mathbb{F}_{p}}^{p q}\right)$ are defined over $\mathbb{F}_{p^{2}}$, and that the action of $\mathrm{Frob}_{p}$ on components and singular points of $X_{\mathbb{F}_{p}}^{p q}$ corresponds to the action of $w_{p}$ on the graph $\mathcal{G}\left(X_{\mathbb{F}_{p}}^{p q}\right)$. Recall that the set of vertices of $\mathcal{G}\left(X_{\mathbb{F}_{p}}^{p q}\right)$ consists of two copies of the set of isomorphism classes of supersingular elliptic curves in characteristic $q$ and that $w_{p}$ sends a vertex from the first copy to the symmetrical vertex from the second. It follows that no vertex of $\mathcal{G}\left(X_{\mathbb{F}_{p}}^{p q}\right)$ is fixed by $w_{p}$. Passing to the quotient curve one sees that the $\mathbb{F}_{p}$-rational components of $X_{\mathbb{F}_{p}}$ are exceptional, and more precisely are obtained by blowing-up the edges $y$ of $\mathcal{G}\left(X_{\mathbb{F}_{p}}\right)$ of length two and such that $w_{p}(y)=-y$ (i.e. the edges which are preserved by $w_{p}$, but whose source and target are permuted). 
As already discussed, those exceptional components arise in two situations, the "cas ramifié" and the "cas non-ramifié" of Ogg. In the first case the lifting of $y$ to $\mathcal{G}\left(X_{\mathbb{F}_{p}}^{p q}\right)$ consists of one edge fixed by $w_{q}$. In the second case $y$ has two preimages in $\mathcal{G}\left(X_{\mathbb{F}_{p}}^{p q}\right)$ exchanged by $w_{q}$, see loc. cit., pp. 204 and 205. Note that the fact that $p q$ is odd implies that $X^{p q}\left(\mathbb{Q}_{p}\right)$ itself is empty: this follows from the point $i$ ) of the Theorem of p. 206 of [21]. The hypotheses of the present lemma, that $\left(\frac{q}{p}\right)=-1$ and the congruences satisfied by $p$ and $q$, imply that we are in the "cas non-ramifié". Let $C_{1}$ and $C_{2}$ be the liftings to $\mathcal{G}\left(X_{\mathbb{F}_{p}}^{p q}\right)$ of a $\mathbb{F}_{p}$-rational component $C_{P_{0}}$ in $\mathcal{G}\left(X_{\mathbb{F}_{p}}\right)$. Using Ribet's description ([24], Section 3) we see that the $C_{i}$ 's correspond to Eichler orders $\Theta$ of level $p$ in $B_{q \infty}$ which contain a fourth root of unity $\zeta_{4}$, so that the length of $C_{i}$ is $\operatorname{card}(\operatorname{Aut}(\Theta / \pm 1))=2$. By Eichler mass formula (e.g. [13], 1.12), as $q \equiv 3 \bmod 4$ there is (up to conjugacy) exactly one maximal order in $B_{q \infty}$ containing $\zeta_{4}$ and, as $p \equiv 1 \bmod 4$, exactly two such orders of level $p$. (One can see this by using Ribet's description according to which $\Theta$ is the endomorphism ring of an "enhanced elliptic curve", i.e. a pair consisting of a supersingular elliptic curve in characteristic $q$ and a cyclic subgroup of order $p$.) Those two orders are switched by $w_{q}$, and this shows the existence and uniqueness of $C_{P_{0}}$. As $C_{P_{0}}$ comes from a blow-up, it intersects other components at two singular points only. To prove the last statement of the lemma we remark that Proposition 2.1 gives the desired identification between $C_{P_{0}}$ and a one-dimensional subtorus $T$. Using again that the Frobenius action on $\mathcal{G}\left(\tilde{X}_{\mathbb{F}_{p}}\right)$ is given by $w_{p}$, and that $w_{p}$ switches the two singular points on $C_{P_{0}}$, we finally obtain that $\operatorname{card}\left(T\left(\mathbb{F}_{p}\right)\right)=p+1$.

Lemma 5.2 If $n$ is a integer such that $\phi(X)(\mathbb{C}) \cap J[n](\mathbb{C}) \neq\{0\}$, then $n \geq(p q) / 245$ provided that $p$ is at least 19 and $q$ is at least 245.

Proof. We first recall that a projective and smooth curve $\mathcal{C}$ over a field $k$ is said to be $n$-gonal over $k$ if there exists a $k$-morphism of degree $n$ from $\mathcal{C}$ to $\mathbb{P}^{1}$. The gonality of $\mathcal{C}$ over $k$ is the smallest $n$ for which $\mathcal{C}$ is $n$-gonal over $k$. In the situation of the lemma, if $\phi(X)(\mathbb{C}) \cap J[n](\mathbb{C})$ contains a non-trivial point $Q$, then $n\left((Q)-\left(P_{0}\right)\right)$ is the divisor of a rational function which provides a morphism of degree $n$ to the projective line, therefore $X$ is $n$-gonal over $\mathbb{C}$. This means that the $\mathbb{C}$-gonality of $X^{p q}$ is at most $2 n$. But according to [1], Theorem 1.1, this gonality is at least $(21 / 200)\left(g\left(X^{p q}\right)-1\right)$. The genus of $X^{p q}$ is given by the following formula ([28], chapter 2; see also [6], Proposition 46 for an immediate formula (note that $p q$ is odd), or [31], remark after Proposition 1.4):

$$
g\left(X^{p q}\right)=1-\frac{1}{4}\left(1-\left(\frac{-1}{p}\right)\right)\left(1-\left(\frac{-1}{q}\right)\right)-\frac{1}{3}\left(1-\left(\frac{-3}{p}\right)\right)\left(1-\left(\frac{-3}{q}\right)\right)+\frac{(p-1)(q-1)}{12}
$$

hence

$$
g\left(X^{p q}\right)-1 \geq-\frac{7}{3}+\frac{(p-1)(q-1)}{12}
$$


An elementary computation now shows that $n \geq \frac{21}{400}\left(g\left(X^{p q}\right)-1\right) \geq \frac{p q}{245}$ provided that $p$ and $q$ are as in the statement.

The two preceding lemmas, together with Lemma 3.5 and Remark 3.6, show that for $q$ and $p$ large enough and verifying $\left(\frac{q}{p}\right)=-1, p \equiv 5 \bmod 12, q \equiv 3 \bmod 4$, all the four hypotheses of Proposition 2.2 are verified with $n=p+1$ : we may therefore speak of the model $X_{p+1}$ of $X_{p+1, \mathbb{Q}_{p}}$ over $\mathbb{Z}_{p}$ provided by Proposition 2.2 .

Theorem 5.3 Keep the above notations and assumptions. Assume that there exists a closed path on $\mathcal{G}(X)$ which is made of Gross vectors and contains, with prime-to- $p$ multiplicity, the edge corresponding to $C_{P_{0}}$. Assume furthermore that $q>245$ and $p \gg q$. Then $P=P_{0}$ and hence $P$ is a special point.

Proof. We choose a basis of $H_{1}(\mathcal{G}(X), \mathbb{Z})$ as in the proof of Proposition 2.1, with only one vector $V_{P_{0}}$ containing (with multiplicity one) the edge corresponding to $C_{P_{0}}$. The path $\gamma$ made of the Gross vectors of the statement has a component at $V_{P_{0}}$ which is nonzero mod $p$, and $\gamma$ corresponds to a character of the winding quotient by Corollary 4.3. We use our basis of $H_{1}(\mathcal{G}(X), \mathbb{Z})$ as in the proof of Proposition 2.1 and pick a $\mathbb{F}_{p}$-parameter $x$ on $C_{P_{0}}$. Let $a$ and $\bar{a}$ be the values taken by $x$ at the singular points of $C_{P_{0}}$, which are $\mathbb{F}_{p^{2}}$-conjugate (Lemma 5.1), and set $\lambda:=\left(x\left(P_{0}\right)-\bar{a}\right) /\left(x\left(P_{0}\right)-a\right)$. With these notations, a point $Q$ in $C_{P_{0}}$ is mapped by $\phi$ to $\left(\lambda \times \frac{x(Q)-a}{x(Q)-\bar{a}}, 1, \ldots, 1\right)$ (Proposition 2.1). This gives the identification via $\phi_{\mathbb{F}_{p}}$ of $C_{P_{0}}$ with the twisted form of $\mathbb{G}_{m}$ over $\mathbb{F}_{p}$ called $T$ in Lemma 5.1. As the fiber of $J$ at $p$ is purely toric, its cotangent space is naturally identified with the tensor product of its character group with $\mathbb{F}_{p}$. We call $\omega_{\gamma}$ the invariant differential on $J_{\mathbb{F}_{p}}$ corresponding to $\gamma$ via this identification. One now computes that, if $\beta$ is the component of $\gamma$ at $V_{P_{0}}$, the pull-back of $\omega_{\gamma}$ to the cotangent space of $X_{\mathbb{F}_{p}}$ at $P_{0}$ is $\beta\left(1 /\left(x\left(P_{0}\right)-a\right)-1 /\left(x\left(P_{0}\right)-\bar{a}\right)\right) d x$, which of course does not vanish. The above shows that $\operatorname{Cot}(\phi)$ is nonzero at $\phi\left(P_{0}\right)$, and this means that $\phi$ is a formal immersion at $P_{0}$. We briefly indicate how one can get the same for $\alpha \circ \phi$, with $\alpha$ defined at the beginning of section 5 (see e.g. the proof of [22], Proposition 3.2). Let $I_{e}^{w_{q}=1}$ be the Eisenstein ideal relative to $J$. The exact sequence of abelian varieties over $\mathbb{Q}$ :

$$
0 \rightarrow I_{e}^{w_{q}=1} \cdot J_{/ \mathbb{Q}} \rightarrow J_{/ \mathbb{Q}} \rightarrow J_{e / \mathbb{Q}}^{w_{q}=1} \rightarrow 0
$$

induces an exact sequence of cotangent spaces at the origin, which are free $\mathbb{Z}_{p}$-modules of finite rank:

$$
0 \rightarrow \operatorname{Cot}\left(J_{e / \mathbb{Z}_{p}}^{w_{q}=1}\right) \rightarrow \operatorname{Cot}\left(J_{/ \mathbb{Z}_{p}}\right) \rightarrow \operatorname{Cot}\left(I_{e}^{w_{q}=1} \cdot J_{/ \mathbb{Z}_{p}}\right) \rightarrow 0
$$

(this follows from the semistability of $J$, see the references in [22], loc. cit.). Using our previous remarks on $\gamma$ and the identification between cotangent spaces and character groups, one 
sees that $\omega_{\gamma}$ actually belongs to the subspace $\operatorname{Cot}\left(J_{e / \mathbb{F}_{p}}^{w_{q}=1}\right)$ of $\operatorname{Cot}\left(J_{/ \mathbb{F}_{p}}\right)$. Therefore the nonvanishing of $\operatorname{Cot}(\phi)\left(\omega_{\gamma}\right)=\operatorname{Cot}(\alpha \circ \phi)\left(\omega_{\gamma}\right)$ shows that $\alpha \circ \phi$ also is a formal immersion at $P_{0}$ (and actually at any point on the component $C_{P_{0}}^{\mathrm{sm}}$ ). As multiplication by an integer $n$ on an abelian variety induces multiplication by $n$ on its cotangent space (with respect to the module structure), one sees that the map $\Psi$ defined at the beginning of this section is a formal immersion at $P_{0}$, because $p+1$ is prime to $p$. Finally, as $\Psi$ factors through $X_{p+1}$, the map $X_{p+1} \rightarrow J_{e}^{w_{q}=1}$ is again a formal immersion at $(p+1) \phi\left(P_{0}\right)\left(\mathbb{F}_{p}\right)$. Furthermore, Lemma 5.1 shows that $(p+1) \phi(P)\left(\mathbb{F}_{p}\right)=0\left(\mathbb{F}_{p}\right)=(p+1) \phi\left(P_{0}\right)\left(\mathbb{F}_{p}\right)$ in $X_{p+1}\left(\mathbb{F}_{p}\right) \hookrightarrow J\left(\mathbb{F}_{p}\right)$.

Summing up: one has two points $(p+1) \phi\left(P_{0}:=w_{p}(P)\right)$ and $(p+1) \phi(P)$ in $X_{p+1}(\mathbb{Q})$, specializing to the same point at $p$, where the natural map to a winding quotient is a formal immersion. This allows us to apply Mazur's classical argument to the curve $X_{p+1}$ (see e.g. [22], proof of the Proposition 3.1 for a version akin to the present one). We therefore obtain that $(p+1)\left(\left(w_{p}(P)\right)-(P)\right)=0$ in $X_{p+1}(\mathbb{Q}) \hookrightarrow J(\mathbb{Q})$. But this means that either $P=w_{p}(P)$, in which case $P$ is a special point, or $X$ is $(p+1)$-gonal. Now Lemma 5.2 shows that the choice of $p$ and $q$ implies that the latter case is impossible.

\section{An explicit infinite family.}

We finally illustrate our method by considering the family of curves $X^{p \cdot 251} / w_{251}$, for $p$ large and running through the infinite set of primes verifying the explicit congruences of Lemma 6.1 below, which ensures that the hypotheses of Theorem 5.3 are verified. Using Gross vectors, we will construct explicit closed paths on the graph $\mathcal{G}:=\mathcal{G}\left(\left(X^{p \cdot 251} / w_{251}\right)_{\mathbb{F}_{p}}\right)$. Call $\mathcal{S}_{1}$ and $\mathcal{S}_{2}$ two copies of the supersingular invariants in characteristic 251. The vertices of $\mathcal{G}$ are the $\operatorname{Gal}\left(\mathbb{F}_{251^{2}} / \mathbb{F}_{251}\right)$-orbits in the $\mathcal{S}_{i}$ s. Denote by $\tilde{\mathcal{G}}:=\mathcal{G}\left(X^{p \cdot 251} / w_{251}\right)$ the desingularized graph. Each $\mathcal{S}_{i}$ is given by the roots of the supersingular polynomial in characteristic 251, which is:

$$
\begin{gathered}
j(j+29)(j+19)(j-64)(j-4)(j-24)(j-30)(j-35)(j-101)(j+112)(j+66)(j+52)(j+44) \times \\
(j+38)\left(j^{2}-60 j-81\right)\left(j^{2}-81 j-68\right)\left(j^{2}-105 j+116\right)\left(j^{2}+93 j+91\right)
\end{gathered}
$$

(see for instance [3]). The second factor of this product is congruent to $(j-1728) \bmod 251$. Let $E_{1728}$ be an elliptic curve over $\mathbb{F}_{251}$ with $j$-invariant 1728 . As $p \equiv 1 \bmod 4$, there are two embeddings $\mathbb{Z}\left[\zeta_{4}\right] \hookrightarrow \operatorname{End}\left(E_{1728}, C_{p}\right)$, for two $p$-isogenies $C_{p}$. They give rise to the two only edges of length 2 in $\mathcal{G}\left(X^{p \cdot 251}\right)$ from the vertex $j=1728$ in $\mathcal{S}_{1}$ to the vertex $j=1728$ in $\mathcal{S}_{2}$ (see the proof of Lemma 5.1). The Gross vector $e_{4}$ in the graph of $X^{p \cdot 251}$ corresponds to the sums of these two edges, which are switched by $w_{251}$, hence $e_{4}$ gives a single edge of length 2 in $\mathcal{G}\left(X^{p \cdot 251} / w_{251}\right)_{\mathbb{F}_{p}}$. In $\tilde{\mathcal{G}}$, this edge finally splits into the two edges (of length 1 ) surrounding the 


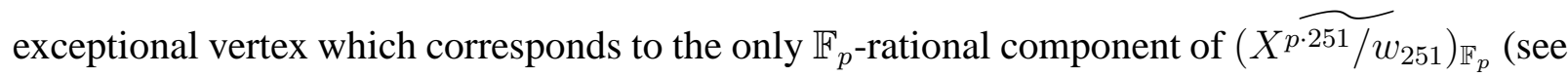
Lemma 5.1).

Now after looking in tables (e.g. [20]) among the Heegner points of degree one and two, one considers the following other three Gross vectors: $e_{28}, e_{36}, e_{267}$, associated to the orders $\mathbb{Z}[\sqrt{-7}]$, $\mathbb{Z}[3 \sqrt{-1}]$ and $\mathbb{Z}[(1+\sqrt{-267}) / 2]$ respectively, with class polynomials:

$$
\begin{gathered}
P_{-28}=(j-16581375) \equiv(j-64) \quad \bmod 251 ; \\
P_{-36}=\left(j^{2}-153542016 * j-1790957481984\right) \equiv(j-64)(j+19) \bmod 251 ; \\
P_{-267}=\left(j^{2}+19683091854079488000000 * j+531429662672621376897024000000\right) \\
\equiv(j+19)(j+29) \quad \bmod 251 .
\end{gathered}
$$

One readily checks that the corresponding quadratic orders embed into the quaternion algebra $B_{251 . \infty}$ (i.e. that 251 is inert in these orders), and if $p$ splits in these orders, then for each one we obtain two $p$-isogenies linking the (one or two) $j$ s of each polynomial. Those isogenies are interpreted as edges from $\mathcal{S}_{1}$ to $\mathcal{S}_{2}$ on the graph $\mathcal{G}\left(X^{p \cdot 251}\right)_{\mathbb{F}_{p}}$, then on $\tilde{\mathcal{G}}$, in the same way as explained in the above case of $e_{4}$. We sum-up the above observations in the following:

Lemma 6.1 Assume that $p$ verifies the following congruence conditions:

$$
p \equiv 5 \bmod 12,\left(\frac{-7}{p}\right)=1,\left(\frac{-267}{p}\right)=1, \text { and }\left(\frac{p}{251}\right)=-1 .
$$

Then the sum of Heegner points: $\left(e_{4}-e_{267}+e_{36}-e_{28}\right)$ defines a closed path in $\mathcal{G}\left(\left(X^{p \cdot 251} / w_{251}\right)_{\mathbb{F}_{p}}\right)$.

Proof. A straightforward application of Theorem 3.1.

Remark 6.2 The path of Lemma 6.1 may have different shapes depending on $p$ - for instance it can have one, two or three connected components. These shapes depend on whether or not the degree-two Heegner points link their two different roots $j$. This in turn is determined by the class of the ideals above $p$ in the Picard group of each corresponding quadratic order, by the theory of complex multiplication for elliptic curves. In any case however, one gets a closed path passing through the edge having $\mathrm{CM}$ by $\zeta_{4}$, which is the only relevant point regarding our problem.

Consider the family of curves $X^{p \cdot 251} / w_{251}$ with $p$ satisfying the congruences above. It follows from Theorem 5.3 that the only possible $\mathbb{Q}$-rational points on the curves of this family are special points. 
Actually, the curves in our family do not have any special rational point either. For a curve $X^{p \cdot 251} / w_{251}$ to have a special rational point, either $\mathbb{Q}(\sqrt{-p})$ or $\mathbb{Q}(\sqrt{-251})$ must have class number one or the field $\mathbb{Q}(\sqrt{-251 p})$ must have class number two (see e.g. [27], section 4). These conditions are not satisfied by the primes $p$ satisfying our conditions.

In general it is difficult to construct infinite families of counter-examples to Hasse principle among the curves of this form (see [27]). However, using a PARI computer program, one can determine, for a given example, whether the curve has local points everywhere. We have done this for a particular example of $p=137$. This prime satisfies the congruences above. Note however that, due to the ineffectiveness of Lemma 3.5, we cannot guarantee the smoothness of the curve $X^{137 \cdot 251} / w_{251}$ along the exceptional $\mathbb{F}_{137}$-rational component. Using the theorem 3.1 of [27], we verify first that the curve $X^{137 \cdot 251} / w_{251}$ has rational points over $\mathbb{R}, \mathbb{Q}_{137}$ and $\mathbb{Q}_{251}$. This is implied by the conditions

1. $\left(\frac{251}{137}\right)=-1$.

2. $251 \equiv 3 \bmod 4$ and $137 \equiv 1 \bmod 4$.

Then one verifies, that for every $\ell<4 g^{2}$ ( $g$ being the genus of the curve) one of the quantities (notations of [27]) $\Sigma_{\ell}(251 \cdot 137)$ or $\Sigma_{251 \ell}(251 \cdot 137)$ is non-zero. This guarantees the existence of local points at all primes of good reduction (i.e. $\ell \neq 137,251$ ). It becomes difficult to verify this condition for primes $p>137$ as one exceeds the PARI limit on precompiled primes.

We state our theorem for an arbitrary $p$.

Theorem 6.3 For every large enough prime $p$ satisfying the congruences of Lemma 6.1 the curve $X^{p \cdot 251} / w_{251}$ does not have $\mathbb{Q}$-rational points.

\section{References}

[1] D. Abramovich, A linear lower bound on the gonality of modular curves. Internat. Math. Res. Notices no. 20 (1996), 1005-1011.

[2] M. Alsina, P. Bayer, Quaternion orders, quadratic forms, and Shimura curves. CRM Monograph Series, 22. AMS, Providence, RI, 2004. xvi+196 pp.

[3] A.O.L. Atkin, Table of supersingular invariants, in Modular functions of one variable, IV (Proc. Internat. Summer School, Univ. Antwerp, Antwerp, 1972), pp. 143-144. Lecture Notes in Math., Vol. 476, Springer, Berlin, 1975.

[4] B. Bollobás, Modern graph theory, GTM n. 184, Springer-Verlag, New York (1998). 
[5] N. Bruin, E. V. Flynn, J. González, V. Rotger, On finiteness conjecture for modular quaternion algebras, Math. Proc. Camb. Phil. Soc. 141:3 (2006), 383-408.

[6] P. Clark, Local and global points on moduli spaces of potentially quaternionic abelian surfaces, Ph. D. Thesis, 2003.

[7] Ch. Cornut, V. Vatsal, Nontriviality of Rankin-Selberg $L$-functions and CM points, preprint (2004), to appear in the Proceedings of a Durham conference.

[8] G. Davidoff, P. Sarnak, A. Valette, Elementary number theory, group theory, and Ramanujan graphs. LMS Student Texts 55. CUP, Cambridge, 2003.

[9] F. Diamond, J. Im, Modular forms and modular curves, in Seminar on Fermat's last theorem (Toronto, 1993-1994), CMS Conf. Proc. no 17, Amer. Math. Soc., Providence, RI, 1995.

[10] B. Edixhoven, Appendix to A rigid analytic Gross-Zagier formula and arithmetic application, by M. Bertolini and H. Darmon, Annals of Maths. 146 (1997), 111-147.

[11] B. Edixhoven, On Néron models, divisors and modular curves, J. Ramanujan Math. Soc. 13 no. 2 (1998), 157-194.

[12] M. Emerton, Supersingular elliptic curves, theta series, and weight two modular forms, J. Amer. Math. Soc., 15 no. 3 (2002), 671-714.

[13] B. Gross, Heights and the special values of $L$-series, Canadian Math. Soc. Conference Proceedings 7, Amer. Math. Soc., Providence (1987), 115-187.

[14] A. Grothendieck, Éléments de géométrie algébrique, IV. Étude locale des schémas et des morphismes de schémas IV. Inst. Hautes Études Sci. Publ. Math. No. 32, 1967, 361 pp.

[15] A. Grothendieck, Séminaire de Géométrie algébrique 7, I, Exposé IX, LNM 288, 313-523, Springer 1972.

[16] H. Jacquet, R.P. Langlands, Automorphic forms on $\mathrm{GL}_{2}$, Lecture Note in Math. no 114, Springer 1970.

[17] B. Jordan, R. Livné, Local Diophantine properties of Shimura curves, Math. Ann. 270 no. 2 (1985), 235-248.

[18] K. Kato, $p$-adic Hodge theory and values of Zeta functions of modular forms, in Cohomologies $p$-adiques et applications arithmétiques, Astérisque n. 295 (2004), ix, 117-290. 
[19] V. A. Kolyvagin, D. Yu. Logachev, Finiteness of the Shafarevich-Tate group and the group of rational points for some modular abelian varieties, Leningrad Math. J., Vol. 1, no. 5 (1990), 1229-1253.

[20] J.-C. Lario, Tables available at http://www-ma2.upc.es/ lario/ellipticm.htm

[21] A. P. Ogg, Mauvaise réduction des courbes de Shimura, in Séminaire de théorie des nombres, Paris 1983-84, 199-217, Progr. Math., 59, Birkhäuser Boston, Boston, MA (1985).

[22] P. Parent, Towards the triviality of $X_{0}^{+}\left(p^{r}\right)(\mathbb{Q})$ for $r>1$, Compositio Math. 141 no. 3 (2005), 561-572.

[23] M. Raynaud, Jacobiennes des courbes modulaires et opérateurs de Hecke, Astérisque 196197 (1991), 9-25.

[24] K. Ribet, On modular representations of $\operatorname{Gal}(\overline{\mathbb{Q}} / \mathbb{Q})$ arising from modular forms, Invent. Math. 100 (1990), no. 2, 431-476.

[25] V. Rotger, Modular Shimura varieties and forgetful maps, Trans. Amer. Math. Soc. 356 (2004), no. 4, 1535-1550.

[26] V. Rotger, Which quaternion algebra act on a modular abelian variety?, preprint (2006).

[27] V. Rotger, A. Skorobogatov, A. Yafaev, Failure of the Hasse principle for Atkin-Lehner quotients of Shimura curves over $\mathbb{Q}$, Moscow Math. Journal vol. 5 (2005) no. 2, 463-476.

[28] G. Shimura, Introduction to the arithmetic theory of automorphic functions. Kanô Memorial Lectures, No. 1. Publications of the Mathematical Society of Japan, No. 11. Iwanami Shoten, Publishers, Tokyo; Princeton University Press, Princeton, N.J., 1971. xiv+267 pp.

[29] A. Skorobogatov, The Brauer-Manin obstruction for Shimura curves, IMRN, to appear.

[30] A. Skorobogatov, S. Siksek, On a Shimura curve that is a counterexample to the Hasse principle, Bull. London Math. Soc. 35 (2003), no. 3, 409-414.

[31] A. Skorobogatov, A. Yafaev, Descent on certain Shimura curves, Israel J. Math. 140 (2004), 319-332.

[32] V. Vatsal, Special value formulae for Rankin $L$-functions. Heegner points and Rankin $L$ series, 165-190, Math. Sci. Res. Inst. Publ., 49, Cambridge Univ. Press, Cambridge, 2004.

[33] Sh.-W. Zhang, Gross-Zagier formula for $\mathrm{GL}_{2}$, Asian J. Math. 5 (2001), 183-290. 
[34] Sh.-W. Zhang, Gross-Zagier formula for $\mathrm{GL}_{2}$ II, in Heegner points and Rankin $L$-series, 191-214, Math. Sci. Res. Inst. Publ. 49, Cambridge Univ. Press, Cambridge, 2004.

Pierre Parent

Institut de Mathématiques de Bordeaux

Université de Bordeaux I

351, cours de la libération

33405 Talence Cédex France

Adresse électronique: Pierre.Parent@math.u-bordeaux1.fr

Andrei Yafaev

Department of Mathematics

University College London

Gower street

London, WC1E 6BT England

E-mail adress: yafaev@math.ucl.ac.uk 